\title{
Some Polygonal Sum Labeling of Paths
}

\author{
S.Murugesan \\ Associate Professor \\ Department of Mathematics \\ CBM college \\ Coimbatore-641042 \\ Tamil Nadu \\ India
}

\author{
D.Jayaraman \\ Associate Professor \\ Department of Mathematics \\ CBM College \\ Coimbatore- 641042 \\ Tamil Nadu \\ India
}

\author{
J.Shiama \\ Assistant Professor \\ Department of Mathematics \\ CMS College of Science and \\ Commerce \\ Coimbatore- 641049 \\ Tamil Nadu \\ India
}

\begin{abstract}
A $(p, q)$ graph $G$ is said to admit a polygonal sum labeling if its vertices can be labeled by non -negative integers such that the induced edge labels obtained by the sum of the labels of end vertices are the first $q$ polygonal numbers. A graph $G$ which admits a polygonal sum labeling is called a polygonal sum graph. In this paper we prove that the paths admit pentagonal, hexagonal, heptagonal, octagonal, nonagonal and decagonal sum labeling. This work is a nice composition of graph theory and combinatorial number theory.
\end{abstract}

\section{INTRODUCTION}

The graphs considered here are finite, connected, undirected and simple. The vertex set and the edge set of a graph $\mathrm{G}$ are denoted by $V(G)$ and $E(G)$ respectively. For various graph theoretic notations and terminology we follow Harary [1] and for number theory we follow Burton [2]. We will give the brief summary of definitions which are useful for the present investigations.

Definition-1.1: If the vertices of the graph are assigned values subject to certain conditions it is known as graph labeling.

A dynamic survey on graph labeling is regularly updated by Gallian [3] and it is published by Electronic Journal of Combinatorics. Vast amount of literature is available on different types of graph labeling and more than 1000 research papers have been published so far in last four decades. Most interesting labeling problems have three important ingredients.

$>$ A set of numbers from which vertex labels are chosen;

$>$ A rule that assigns a value to each edge;

$>$ A condition that these values must satisfy.

The present work is aimed to discuss one such labeling known as polygonal sum labeling.

Definition-1.2: Polygonal numbers are just the number of vertices in a figure formed by a certain polygon. The first number in any group of polygonal numbers is always one or a point. The second number is equal to the number of vertices of the polygon. The third number is made by extending two of the sides of the polygon from the second polygonal number, completing the larger polygon and placing vertices and other points wherever necessary. The third polygonal number is found by adding all the vertices and points in the resulting figure.
Triangular Numbers
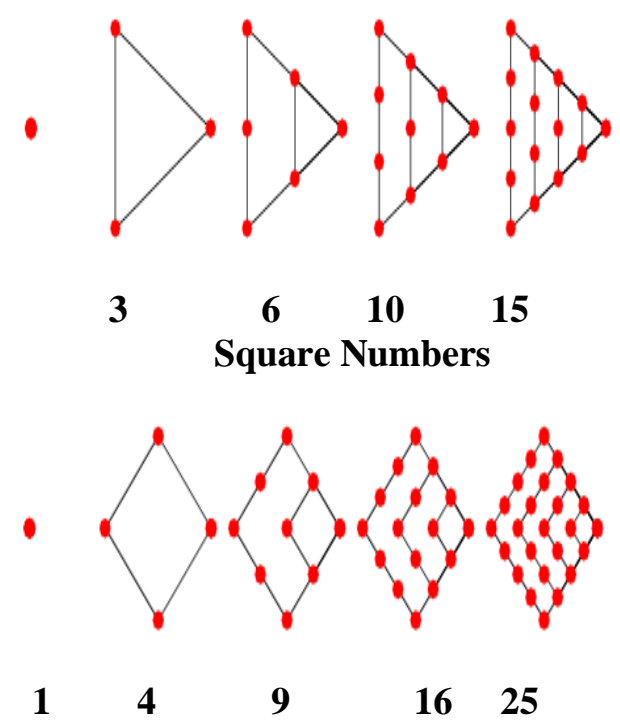

Definition-1.3: Pentagonal numbers are numbers that create a pentagon. In other words: $1,5,12,22,35,51,70,92$, $117,145, \ldots$

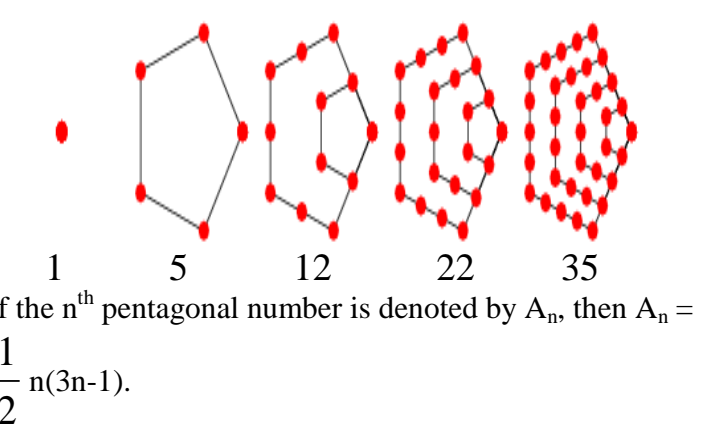

Definition-1.4: A pentagonal sum labeling of a graph $\mathrm{G}$ is one to one function $f: V(G) \rightarrow N$ (where $N$ is the set of all non- negative integers) that induces a bijection $\mathrm{f}^{+}: \mathrm{E}(\mathrm{G}) \rightarrow$ $\left\{A_{1}, A_{2}, \ldots, A_{q}\right\}$ of the edges of $G$ defined by $f^{+}(u v)=f(u)+$ $f(v)$ for every $e=u v \in E(G)$. The graph which admits such labeling is called a pentagonal sum graph. 
Example-1.5: A pentagonal sum graph

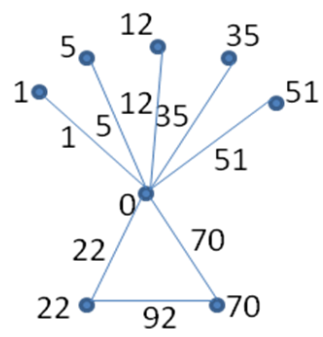

Definition-1.6: Hexagonal numbers are numbers that create a hexagon. In other words: $1,6,15,28,45,66,91,120$, $153,190,231,276$,

\section{0}

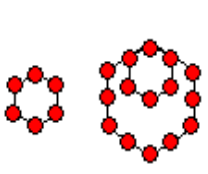

16

15

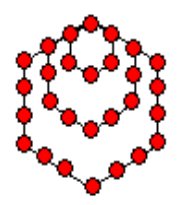

28

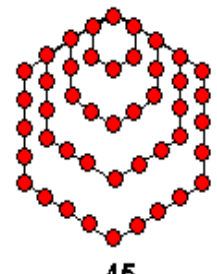

45
If the $\mathrm{n}^{\text {th- }}$ hexagonal number is denoted by $B_{n}$, then $B_{n}=n(2 n$ $1)$.

Definition-1.7: A hexagonal sum labeling of a graph $\mathrm{G}$ is a one-to-one function $\mathrm{f}: \mathrm{V}(\mathrm{G}) \rightarrow \mathrm{N}$ that induces a bijection $\mathrm{f}^{+}$: $\mathrm{E}(\mathrm{G}) \rightarrow\left\{\mathrm{B}_{1}, \mathrm{~B}_{2}, \ldots, \mathrm{B}_{\mathrm{q}}\right\}$ of the edges of $\mathrm{G}$ defined by $\mathrm{f}^{+}(\mathrm{uv})$ $=\mathrm{f}(\mathrm{u})+\mathrm{f}(\mathrm{v})$ for every $\mathrm{e}=\mathrm{uv} \in \mathrm{E}(\mathrm{G})$. The graph which admits such labeling is called a hexagonal sum graph.

\section{Example-1.8:}

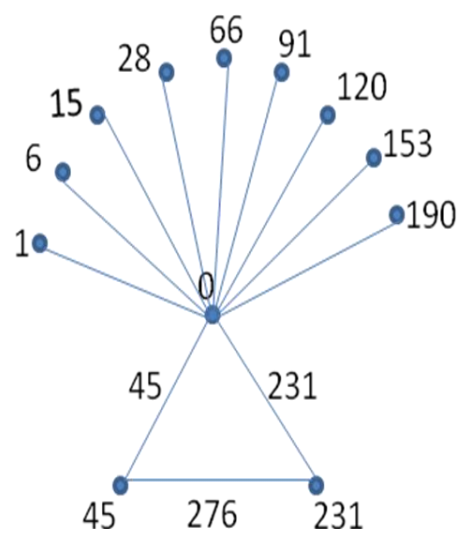

Definition-1.9: Heptagonal numbers are numbers that create a heptagon. In other words: $1,7,18,34,55,81,112$, $148,189,235,286,342,403,469,540,616,697, \ldots$

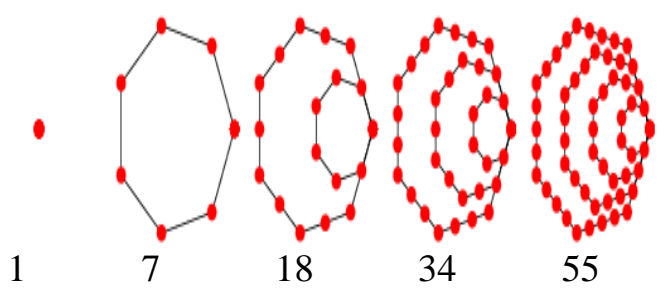

In the $\mathrm{n}^{\text {th }}$ heptagonal number is denoted by $\mathrm{C}_{\mathrm{n}}$, then $\mathrm{C}_{\mathrm{n}}=\frac{1}{2}$ $\mathrm{n}(5 \mathrm{n}-3)$.

Definition-1.10: A heptagonal sum labeling of a graph $\mathrm{G}$ is a one-to-one function $\mathrm{f}: \mathrm{V}(\mathrm{G}) \rightarrow \mathrm{N}$ that induces a bijection $\mathrm{f}^{+}: \mathrm{E}(\mathrm{G}) \rightarrow\left\{\mathrm{C}_{1}, \mathrm{C}_{2}, \ldots, \mathrm{C}_{\mathrm{q}}\right\}$ of the edges of $\mathrm{G}$ defined by $\mathrm{f}^{+}(\mathrm{uv})=\mathrm{f}(\mathrm{u})+\mathrm{f}(\mathrm{v})$ for every $\mathrm{e}=\mathrm{uv} \in \mathrm{E}(\mathrm{G})$. The graph which admits such labeling is called a heptagonal sum graph.

\section{Example-1.11:}

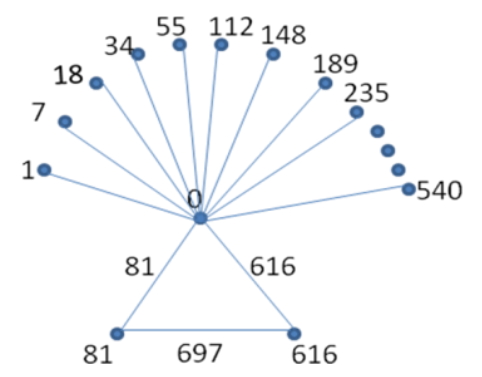

Definition-1.12: Octagonal numbers are numbers that create an octagon. In other words: $1,8,21,40,65,96$, $133,176,225,280,341,408,481,560,645,736,833,936,1045$, $1160,1281,1408,1541, \ldots$

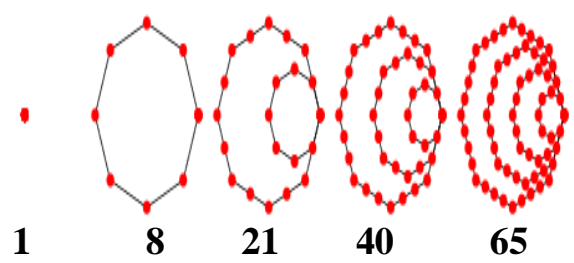

In the $\mathrm{n}^{\text {th }}$ octagonal number is denoted by $D_{n}$, then $D_{n}=n(3 n-$ 2).

Definition-1.13: An octagonal sum labeling of a graph $G$ is a one-to-one function $\mathrm{f}: \mathrm{V}(\mathrm{G}) \rightarrow \mathrm{N}$ that induces a bijection $f^{+}: E(G) \rightarrow\left\{D_{1}, D_{2}, \ldots, D_{q}\right\}$ of the edges of $G$ defined by $\mathrm{f}^{+}(\mathrm{uv})=\mathrm{f}(\mathrm{u})+\mathrm{f}(\mathrm{v})$ for every $\mathrm{e}=\mathrm{uv} \in \mathrm{E}(\mathrm{G})$. The graph which admits such labeling is called an octagonal sum graph.

Example-1.14:

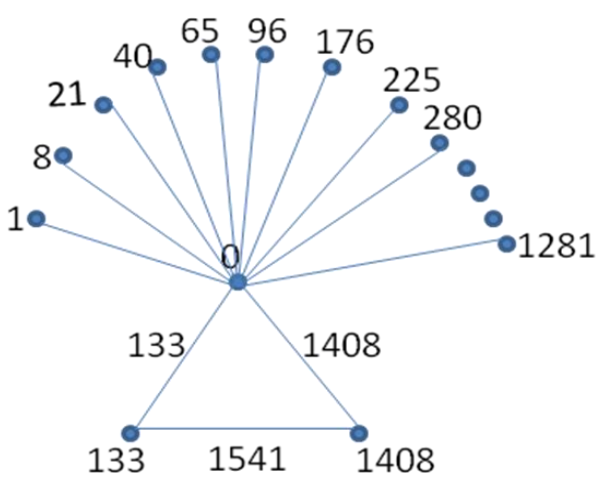

Definition-1.15: Nonagonal numbers are numbers that create a nonagon. In other words: $1,9,24,46,75,111,154$, 204, 261, 325, 396, 474, 559, 651, 750, 856, 969, 1089, 1216, 1350, 1491, 1639, 1794,1956, 2125, 2301, 2484, 2674, 2871, $3075, \ldots$ 


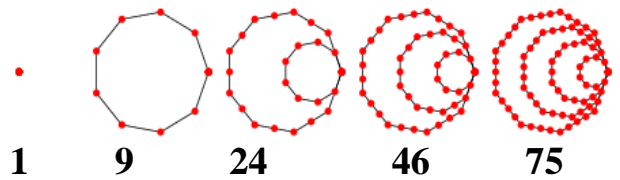

In the $\mathrm{n}^{\text {th }}$ nonagonal number is denoted by $\mathrm{E}_{\mathrm{n}}$, then $\mathrm{E}_{\mathrm{n}}$ $=\frac{1}{2} \mathrm{n}(7 \mathrm{n}-5)$.

Definition-1.16: A nonagonal sum labeling of a graph $\mathrm{G}$ is a one-to-one function $\mathrm{f:V}(\mathrm{G}) \rightarrow \mathrm{N}$ that induces a bijection $\mathrm{f}^{+}: \mathrm{E}(\mathrm{G}) \rightarrow\left\{\mathrm{E}_{1}, \mathrm{E}_{2}, \ldots, \mathrm{E}_{\mathrm{q}}\right\}$ of the edges of $\mathrm{G}$ defined by $\mathrm{f}^{+}(\mathrm{uv})=\mathrm{f}(\mathrm{u})+\mathrm{f}(\mathrm{v})$ for every $\mathrm{e}=\mathrm{uv} \in \mathrm{E}(\mathrm{G})$. The graph which admits such labeling is called a nonagonal sum graph.

\section{Example-1.17:}

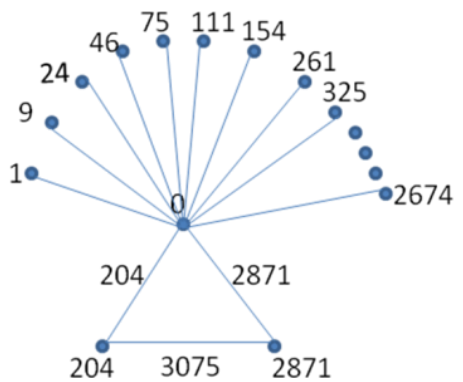

Definition-1.18: Decagonal numbers are numbers that create a decagon. In other words:

$1,10,27,52,85,126,175,232,297,370,451,540,637,742$,

$855,976,1105,1242,1387,1540,1701,1870,2047,2232$,

$2425,2626,2835,3052,3277,3510,3751,4000,4257$,

$4522,4795,5076,5365,5662, \ldots$

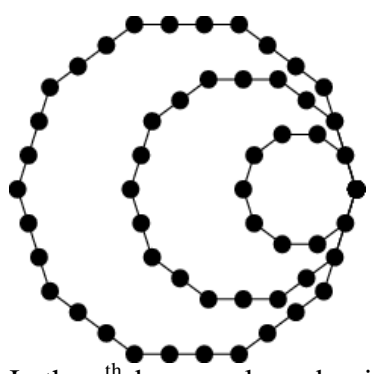

In the $\mathrm{n}^{\text {th }}$ decagonal number is denoted by $\mathrm{F}_{\mathrm{n}}$, then $\mathrm{F}_{\mathrm{n}}=\mathrm{n}(4 \mathrm{n}$ 3).

Definition-1.19: A decagonal sum labeling of a graph $\mathrm{G}$ is a one-to-one function $\mathrm{f:V}(\mathrm{G}) \rightarrow \mathrm{N}$ that induces a bijection $\mathrm{f}^{+}: \mathrm{E}(\mathrm{G}) \rightarrow\left\{\mathrm{F}_{1}, \mathrm{~F}_{2}, \ldots, \mathrm{F}_{\mathrm{q}}\right\}$ of the edges of $\mathrm{G}$ defined by $\mathrm{f}^{+}(\mathrm{uv})=\mathrm{f}(\mathrm{u})+\mathrm{f}(\mathrm{v})$ for every $\mathrm{e}=\mathrm{uv} \in \mathrm{E}(\mathrm{G})$. The graph which admits such labeling is called a decagonal sum graph.

\section{Example-1.20:}

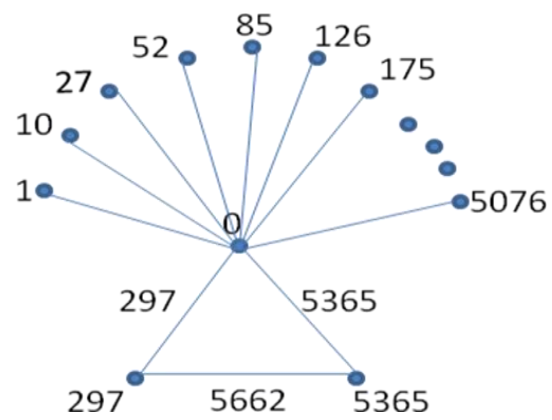

\section{MAIN RESULTS}

Here we prove that the paths $\mathrm{P}_{\mathrm{n}}$ admit pentagonal, hexagonal, heptagonal, octagonal, nonagonal and decagonal sum labeling.

Theorem 2.1: The path $P_{n}$ admits pentagonal sum labeling. Proof: Let $P_{n}: u_{1} u_{2} u_{3} \ldots u_{n}$ be the path and $v_{i=} u_{i} u_{i+1}(1 \leq i \leq$ $\mathrm{n}-1)$ be the edges.

For $\mathrm{i}=1,2, \ldots, \mathrm{n}$, define

$$
\begin{aligned}
f\left(u_{i}\right) & =\frac{1}{4}(i-1)(3 i-1) \text { if } i \text { is odd } \\
& =\frac{1}{4} i(3 i-4) \quad \text { if } i \text { is even }
\end{aligned}
$$

We will prove that the induced edge labels obtained by the sum of the labels of end vertices are the first n-1 pentagonal numbers.

$$
\begin{aligned}
& \text { Case }(\mathrm{i}) \mathrm{i} \text { is odd } \\
& \mathrm{f}\left(\mathrm{u}_{\mathrm{i}}\right)+\mathrm{f}\left(\mathrm{u}_{\mathrm{i}+1}\right)= \\
& \begin{aligned}
\frac{1}{4}(i-1)(3 i-1)+\frac{1}{4}(i+1)[3(i+1)-4] \\
=\frac{1}{4}(i-1)(3 i-1)+\frac{1}{4}(i+1)(3 i-1) \\
=\frac{1}{2}(i)(3 i-1) \\
=\mathrm{A}_{\mathrm{i}} \\
=\mathrm{f}^{+}\left(\mathrm{v}_{\mathrm{i}}\right)
\end{aligned}
\end{aligned}
$$

Case(ii) $\mathrm{i}$ is even

$$
\begin{aligned}
\mathrm{f}\left(\mathrm{u}_{\mathrm{i}}\right)+\mathrm{f}\left(\mathrm{u}_{\mathrm{i}+1}\right) & =\frac{1}{4}(i)(3 i-4)+\frac{1}{4}(i)(3 i+2) \\
& =\frac{1}{2}(i)(3 i-1) \\
& =\mathrm{A}_{\mathrm{i}} \\
& =\mathrm{f}^{+}\left(\mathrm{v}_{\mathrm{i}}\right)
\end{aligned}
$$

Thus the induced edge labels are the first $\mathrm{n}-1$ pentagonal numbers.

Hence $\mathrm{P}_{\mathrm{n}}$ admits pentagonal sum labeling.

Example2.2: The pentagonal sum labeling of $\mathrm{P}_{11}$ is shown in the following figure.

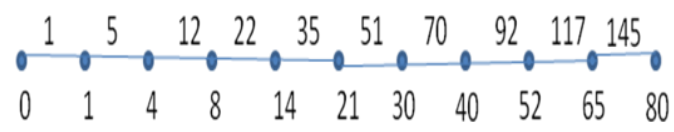

Theorem-2.3: The path $P_{n}$ admits hexagonal sum labeling. Proof: Let $P_{n}: u_{1} u_{2} u_{3} \ldots u_{n}$ be the path and $\quad v_{i}=u_{i} u_{i+1}$ $(1 \leq \mathrm{i} \leq \mathrm{n}-1)$ be the edges.

For $\mathrm{i}=1,2, \ldots, \mathrm{n}$, define

$$
\mathrm{f}\left(\mathrm{u}_{\mathrm{i}}\right)=\frac{1}{2}(i-1)(2 i-1) \text { if } \mathrm{i} \text { is odd }
$$




$$
=\frac{1}{2}(i)(2 i-3) \text { if } \mathrm{i} \text { is even }
$$

Case(i) $i$ is odd

$$
\begin{aligned}
\mathrm{f}\left(\mathrm{u}_{\mathrm{i}}\right)+\mathrm{f}\left(\mathrm{u}_{\mathrm{i}+1}\right) & =\frac{1}{2}(i-1)(2 i-1)+\frac{1}{2}(i+1)(2 i-1) \\
& =(i)(2 i-1) \\
& =\mathrm{B}_{\mathrm{i}} \\
& =\mathrm{f}^{+}\left(\mathrm{v}_{\mathrm{i}}\right)
\end{aligned}
$$

Case(ii) $\mathrm{i}$ is even

$$
\begin{aligned}
\mathrm{f}\left(\mathrm{u}_{\mathrm{i}}\right)+\mathrm{f}\left(\mathrm{u}_{\mathrm{i}+1}\right) & =\frac{1}{2}(i)(2 i-3)+\frac{1}{2}(i)(2 i+1) \\
& =(i)(2 i-1) \\
& =\mathrm{B}_{\mathrm{i}} \\
& =\mathrm{f}^{+}\left(\mathrm{v}_{\mathrm{i}}\right)
\end{aligned}
$$

Thus the induced edge labels are the first $n-1$ hexagonal numbers.

Hence $P_{n}$ admits hexagonal sum labeling.

Example2.4: The hexagonal sum labeling of $P_{10}$ is shown below.

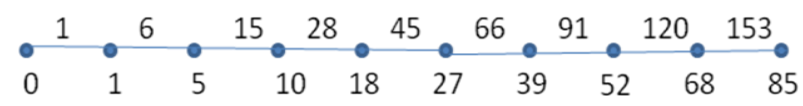

Theorem-2.5: The path $\mathrm{P}_{\mathrm{n}}$ admits heptagonal sum labeling.

Proof: Let $P_{n}: u_{1} u_{2} u_{3} \ldots u_{n}$ be the path and $v_{i=} u_{i} u_{i+1}(1 \leq$ $\mathrm{i} \leq \mathrm{n}-1)$ be the edges.

For $\mathrm{i}=1,2, \ldots, \mathrm{n}$, define

$$
\begin{aligned}
\mathrm{f}\left(\mathrm{u}_{\mathrm{i}}\right) & =\frac{1}{4}(i-1)(5 i-3) \text { if } \mathrm{i} \text { is odd } \\
& =\frac{1}{4}(i)(5 i-8) \quad \text { if } \mathrm{i} \text { is even }
\end{aligned}
$$

Case(i) $i$ is odd

$$
\begin{aligned}
\mathrm{f}\left(\mathrm{u}_{\mathrm{i}}\right)+\mathrm{f}\left(\mathrm{u}_{\mathrm{i}+1}\right) & =\frac{1}{4}(i-1)(5 i-3)+\frac{1}{4}(i+1)(5 i-3) \\
& =\frac{1}{2}(i)(5 i-3) \\
& =\mathrm{C}_{\mathrm{i}} \\
& =\mathrm{f}^{+}\left(\mathrm{v}_{\mathrm{i}}\right)
\end{aligned}
$$

Case(ii) $\mathrm{i}$ is even

$$
\begin{aligned}
\mathrm{f}\left(\mathrm{u}_{\mathrm{i}}\right)+\mathrm{f}\left(\mathrm{u}_{\mathrm{i}+1}\right) & =\frac{1}{4}(i)(5 i-8)+\frac{1}{4}(i)(5 i+2) \\
& =\frac{1}{2}(i)(5 i-3) \\
& =\mathrm{C}_{\mathrm{i}} \\
& =\mathrm{f}^{+}\left(\mathrm{v}_{\mathrm{i}}\right)
\end{aligned}
$$

Thus the induced edge labels are the first $\mathrm{n}-1$ heptagonal numbers.

Hence $\mathrm{P}_{\mathrm{n}}$ admits heptagonal sum labeling.

Example2.6: The heptagonal sum labeling of $\mathrm{P}_{9}$ is shown below

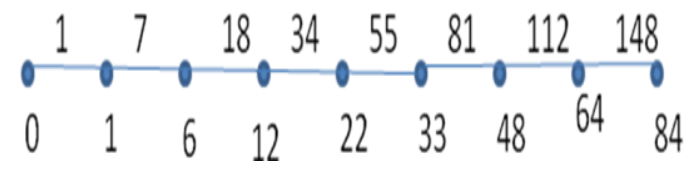

Theorem-2.7: The path $\mathrm{P}_{\mathrm{n}}$ admits octagonal sum labeling. Proof: Let $P_{n}: u_{1} u_{2} u_{3} \ldots u_{n}$ be the path and $v_{i=} u_{i} u_{i+1}(1 \leq i \leq$ n-1) be the edges.

For $\mathrm{i}=1,2, \ldots, \mathrm{n}$, define

$$
\begin{aligned}
\mathrm{f}\left(\mathrm{u}_{\mathrm{i}}\right) & =\frac{1}{2}(i-1)(3 i-2) \text { if } \mathrm{i} \text { is odd } \\
& =\frac{1}{2}(i)(3 i-5) \quad \text { if } \mathrm{i} \text { is even }
\end{aligned}
$$

Case(i) $\mathrm{i}$ is odd

$$
\begin{aligned}
\mathrm{f}\left(\mathrm{u}_{\mathrm{i}}\right)+\mathrm{f}\left(\mathrm{u}_{\mathrm{i}+1}\right) & =\frac{1}{2}(i-1)(3 i-2)+\frac{1}{2}(i+1)(3 i-2) \\
& =(i)(3 i-2) \\
& =\mathrm{D}_{\mathrm{i}} \\
= & \mathrm{f}^{+}\left(\mathrm{v}_{\mathrm{i}}\right)
\end{aligned}
$$

Case(ii) $\mathrm{i}$ is even

$$
\begin{aligned}
\mathrm{f}\left(\mathrm{u}_{\mathrm{i}}\right)+\mathrm{f}\left(\mathrm{u}_{\mathrm{i}+1}\right) & =\frac{1}{2}(i)(3 i-5)+\frac{1}{2}(i)(3 i+1) \\
& =(i)(3 i-2) \\
& =\mathrm{D}_{\mathrm{i}} \\
& =\mathrm{f}^{+}\left(\mathrm{v}_{\mathrm{i}}\right)
\end{aligned}
$$

Thus the induced edge labels are the first n-1 octagonal numbers.

Hence $P_{n}$ admits octagonal sum labeling.

Example2.8: The octagonal sum labeling of $P_{12}$ is shown below.

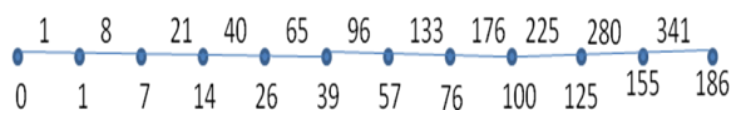

Theorem-2.9: The path $\mathrm{P}_{\mathrm{n}}$ admits nonagonal sum labeling.

Proof: Let $P_{n}: u_{1} u_{2} u_{3} \ldots u_{n}$ be the path and $v_{i=} u_{i} u_{i+1}(1 \leq i \leq$ $\mathrm{n}-1)$ be the edges. For $\mathrm{i}=1,2, \ldots, \mathrm{n}$, define

$$
\mathrm{f}\left(\mathrm{u}_{\mathrm{i}}\right)=\frac{1}{4}(i-1)(7 i-5) \text { if } \mathrm{i} \text { is odd }
$$




$$
=\frac{1}{4}(i)(7 i-12) \quad \text { if } i \text { is even }
$$

Case(i) $i$ is odd

$$
\begin{aligned}
\mathrm{f}\left(\mathrm{u}_{\mathrm{i}}\right)+\mathrm{f}\left(\mathrm{u}_{\mathrm{i}+1}\right) & =\frac{1}{4}(i-1)(7 i-5)+\frac{1}{4}(i+1)(7 i-5) \\
& =\frac{1}{2}(i)(7 i-5) \\
& =\mathrm{E}_{\mathrm{i}} \\
& =\mathrm{f}^{+}\left(\mathrm{v}_{\mathrm{i}}\right)
\end{aligned}
$$

Case(ii) $\mathrm{i}$ is even

$$
\begin{aligned}
\mathrm{f}\left(\mathrm{u}_{\mathrm{i}}\right)+\mathrm{f}\left(\mathrm{u}_{\mathrm{i}+1}\right) & =\frac{1}{4}(i)(7 i-12)+\frac{1}{4}(i)(7 i+2) \\
& =\frac{1}{2}(i)(7 i-5) \\
& =\mathrm{E}_{\mathrm{i}} \\
& =\mathrm{f}^{+}\left(\mathrm{v}_{\mathrm{i}}\right)
\end{aligned}
$$

Thus the induced edge labels are the first n-1 nonagonal numbers.

Hence $P_{n}$ admits nonagonal sum labeling.

Example2.10: The nonagonal sum labeling of $P_{11}$ is shown below

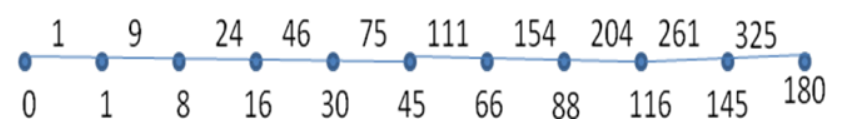

Theorem-2.11:The path $\mathrm{P}_{\mathrm{n}}$ admits decagonal sum labeling.

Proof: Let $P_{n}: u_{1} u_{2} u_{3} \ldots u_{n}$ be the path and $v_{i=} u_{i} u_{i+1}(1 \leq$ $\mathrm{i} \leq \mathrm{n}-1)$ be the edges.

For $\mathrm{i}=1,2, \ldots, \mathrm{n}$, define

$$
\begin{aligned}
\mathrm{f}\left(\mathrm{u}_{\mathrm{i}}\right) & =\frac{1}{2}(i-1)(4 i-3) \text { if } \mathrm{i} \text { is odd } \\
& =\frac{1}{2}(i)(4 i-7) \quad \text { if } \mathrm{i} \text { is even }
\end{aligned}
$$

Case(i) $i$ is odd

$$
\begin{gathered}
\mathrm{f}\left(\mathrm{u}_{\mathrm{i}}\right)+\mathrm{f}\left(\mathrm{u}_{\mathrm{i}+1}\right)= \\
\frac{1}{2}(i-1)(4 i-3)+\frac{1}{2}(i+1)(4 i-3) \\
=(i)(4 i-3) \\
=\mathrm{F}_{\mathrm{i}} \\
=\mathrm{f}^{+}\left(\mathrm{v}_{\mathrm{i}}\right)
\end{gathered}
$$

Case(ii) $\mathrm{i}$ is even

$$
\begin{aligned}
\mathrm{f}\left(\mathrm{u}_{\mathrm{i}}\right)+\mathrm{f}\left(\mathrm{u}_{\mathrm{i}+1}\right) & =\frac{1}{2}(i)(4 i-7)+\frac{1}{2}(i)(4 i+1) \\
& =(i)(4 i-3) \\
& =\mathrm{F}_{\mathrm{i}} \\
& =\mathrm{f}^{+}\left(\mathrm{v}_{\mathrm{i}}\right)
\end{aligned}
$$

Thus the induced edge labels are the first $n-1$ decagonal numbers.

Hence $\mathrm{P}_{\mathrm{n}}$ admits decagonal sum labeling.

Example2.12: The decagonal sum labeling of $\mathrm{P}_{10}$ is shown below

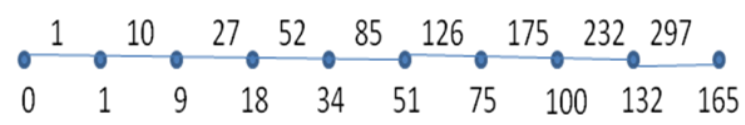

\section{Conclusion}

The polygonal sum labeling can be verified for many other graphs. Also some more polygonal sum labeling can be investigated.

\section{REFERENCES}

[1] Frank Harary, Graph theory, Narosa Publishing House(2001).

[2] D.M.Burton, Elementary number theory, Brown publishers, Second edition(1990)

[3] J A Gallian, A dynamic survey of graph labeling, The Electronic journal of Combinatorics, 17(2010) \# DS6

[4] S.K.Vaidya, U.M.Prajapatti and P.L.Vihol, some Important Results on Triangular sum graphs, Applied Mathematical Sciences, Vol.3,2009,no.36,1763-1772 